\title{
Stress relaxation and misfit dislocation nucleation in the growth of misfitting films: A molecular dynamics simulation study
}

\author{
Liang Dong, Jurgen Schnitker, Richard W. Smith, a) and David J. Srolovitz ${ }^{\text {b) }}$ \\ Department of Materials Science and Engineering, University of Michigan, Ann Arbor, \\ Michigan 48109-2136
}

(Received 7 April 1997; accepted for publication 28 September 1997)

\begin{abstract}
The low-temperature growth and relaxation of misfitting films are analyzed on the basis of two-dimensional molecular dynamics simulations using Lennard-Jones potentials. The temporal evolution of the surface morphology and the mechanisms for misfit dislocation nucleation and stress relaxation are monitored. Pseudomorphic film growth is observed up to a critical thickness. In some cases, the formation of voids within the film relaxes some of the stress. At the critical thickness, dislocations nucleate and relax most of the misfit. The critical thickness increases with decreasing lattice mismatch and depends on the sign of the misfit. The critical thickness of compressively strained films is smaller than that of films with the same magnitude of misfit, but in tension. The mechanism of dislocation nucleation is different in tension and compression and, in all cases, is associated with the roughness of the film surface. In the compressive misfit case, dislocations nucleate by squeezing-out an atom at the base of surface depressions. In the tensile misfit case, however, the nucleation of misfit dislocations involves the concerted motion of a relatively large number of atoms, leading to insertion of an extra lattice (plane) row into an already continuous film. These results show that the critical thickness depends intimately on the film morphology which, in turn, is determined as an integral part of the film growth process. (C) 1998 American Institute of Physics. [S0021-8979(98)05401-2]
\end{abstract}

\section{INTRODUCTION}

In the initial stages of growth of a film which is misfitting with respect to the substrate, the deposited layers are commonly strained into particular crystallographic relationships with respect to the substrate. As the film thickness increases, the elastic energy stored within the film increases. At some thickness, it becomes energetically favorable to relieve this misfit strain by the formation of dislocations at or near the substrate/film interface. These dislocations destroy the coherency of the interface and, therefore, increase its energy. It is when the elastic energy relieved by the dislocations balances the increase in interfacial energy associated with their formation that it becomes thermodynamically favorable to introduce dislocations at the interface. The film thickness at which this occurs is known as the critical thickness.

Several models have been proposed in order to determine the critical thickness as a function of materials parameters. The earliest such treatment was the continuum theory of Frank and van der Merwe for an array of noninteracting dislocations at the film-substrate interface. ${ }^{1}$ Using isotropic elastic analysis, they determined the critical thickness via the type of energy balance described above. This approach does not consider the mechanism by which the dislocations are nucleated.

Matthews and Blakeslee ${ }^{2}$ were the first to examine how the dislocation nucleation mechanism influences the critical thickness. Preexisting dislocations in the substrate are assumed to move into the film once the mean stress caused by

\footnotetext{
a)Present address: 2403 Corteland Dr., Pittsburgh, PA 15241.

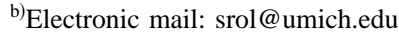

the misfit strain exceeds the dislocation line tension. This results in a force balance for the threading dislocations, which was used to predict the critical thickness. This approach yields critical thicknesses which are identical ${ }^{3}$ to those found in the thermodynamic approach of Frank and van der Merwe. ${ }^{1}$

Experimentally determined critical thicknesses are usually much larger than the values calculated using these theoretical approaches. ${ }^{4,5}$ Part of the explanation of this discrepancy may be attributed to spurious experimental thickness measurements associated with the finite spatial resolution ${ }^{6}$ of the measurements. It is generally believed, however, that there are inherent flaws in these simple continuum models, such as the neglect of dislocations away from the interface or the neglect of kinetic barriers for dislocation nucleation. Accordingly, several modified theories have been proposed. ${ }^{6-9}$

People and Bean assumed that misfit dislocations form when the strain energy density of the film exceeds the selfenergy of an isolated screw dislocation at a fixed distance from the free surface. ${ }^{7}$ Measurements of the critical thickness of $\mathrm{Ge}_{x} \mathrm{Si}_{1-x}$ films on $\mathrm{Si}$ are reportedly better reproduced with this model than with any previous model. Fritz modified the Matthews and Blakeslee theory by scaling the number of dislocations that are present for thickness values greater than the critical thickness, thus making it possible to account for the effects of the finite spatial resolution of the experimental data. ${ }^{6}$ The model predicts fewer misfit dislocations than are obtained in the earlier equilibrium calculations, in agreement with the experiment. Dodson and Tsao considered a kinetic model for the relaxation and dislocation dynamics of a metastable strained layer that is initially coherent. ${ }^{8}$ The model led the authors to suggest that it is the sluggishness of the initial relaxation of the misfit strain that allows pseudomorphic film 
growth to much greater thickness than predicted by the equilibrium theories. Kamat and Hirth used a continuum theory to compare dislocation nucleation in the interior of a strained multilayer structure with nucleation on singular and vicinal surfaces. ${ }^{9}$ They found that nucleation on a vicinal surface is most favorable. Most importantly, it was determined that strained, misfitting films are stable against dislocation injection even when the thickness is equal to the MatthewsBlakeslee critical value, in contradiction to the assumptions of the theory ${ }^{2}$ mentioned above.

Thus, despite some encouraging advances, there is at this time no unified and comprehensive theory of misfit relaxation in strained films. Nevertheless, there is widespread agreement on the point that purely thermodynamic approaches (like that of Frank and van der Merwe) are insufficient for predicting the critical thickness for relaxation. ${ }^{6-9}$ Therefore, an explicitly kinetic theory and a mechanistic understanding of misfit dislocation nucleation are clearly essential. Further, past critical thickness theories have all been based upon continuum analyses. It is very likely that atomic scale phenomena play a central role in determining the critical thickness through their influence on dislocation nucleation phenomena. Because of these limitations in the theories for critical thickness, it is prudent to employ atomistic simulations to help identify physical phenomena that have not, heretofore, been adequately addressed by theory.

While many atomistic simulations of the growth of unstrained films have been performed, there have been relatively few atomistic studies of strained substrate-film systems (e.g., Refs. 10-16). Many of these studies did not involve full dynamical simulations of the growth process. Nandedkar, ${ }^{12}$ e.g., studied misfit dislocations in the bimetallic $\mathrm{Au}$ on Ni system by minimizing the strain energy. The results were interpreted to imply that the nucleation of misfit dislocations starts with the formation of vacancies at the film surface. As the vacancies migrate toward the substrate, a dislocation loop moves toward the interface. Cohen-Solal et $a .^{13}$ developed an interfacial misfit dislocation model based on simulations with Keating's valence force field approximation. The critical thickness values for $\mathrm{CdTe}$ on $\mathrm{CdZnTe}$ as well as for GeSi on $\mathrm{Si}$ they obtained were in good agreement with the experimental data. Interestingly, a simple power law for the critical thickness $h_{c}$ dependence on the lattice mismatch $\eta, h_{c} \sim \eta^{-3 / 2}$ was shown to provide a good fit to the simulation data. ${ }^{13}$

Ichimura and Narayan used a Stillinger-Weber-type potential to investigate semiconductor heterostructures ${ }^{14}$ and also considered the specific role of surface steps. ${ }^{15}$ The calculated critical thickness values they obtained were larger than those predicted using the classical continuum models. They attributed this discrepancy to the dislocation core energy. ${ }^{14}$ They observed an energetic barrier associated with the propagation of a dislocation loop from the surface towards the interface, which they associated with the activation barrier for misfit dislocation nucleation. ${ }^{14}$ Interestingly, they found that the critical thickness depends on the sign of the misfit. If the coherent film is in tension (negative misfit) the critical thickness was found to be larger than when the film is in compression (positive misfit). ${ }^{15}$ They associated this be- havior with the stress dependence of the surface energy.

All of the theoretical assessments of the critical thickness described above were based upon assumptions of a flat (or nearly flat) surface. It is well known that atomically flat surfaces are a rarity in almost any type of coherent film growth. The actual surface morphologies depend on a wide range of kinetic phenomena that come into play during film growth. Therefore, it is appropriate to examine the nature of the critical thickness simultaneously with film growth.

In the present work, we use dynamic, atomic scale simulations of the growth of misfitting films to examine the atomic-level mechanism of dislocation nucleation. This study follows up some preliminary work that we reported earlier. ${ }^{17}$ We perform a series of molecular dynamics (MD) film growth simulations over a wide range of film-substrate misfits to thicknesses that exceed the predicted critical thicknesses. The MD simulation results for the critical thickness are explicitly compared with thermodynamic (i.e., static relaxation) calculations of the critical thickness using exactly the same interatomic potentials.

For the sake of clarity and the computational efficiency required to perform a large number of simulations, we chose to perform this study using a very simple model system: i.e., a two-dimensional simulation in which the atoms interact through simple Lennard-Jones-type pair potentials. This type of interatomic potential provides a reasonable basis for modeling close packed solids. It should be noted that this model does not represent any particular material in detail. Nor is it directly applicable to nonclose packed materials, i.e., most semiconductors and ceramics. Comparison of the present simulation results with those obtained using more refined potentials would be very instructive and should be the subject of future studies.

The simulations were all carried out using a thermostated MD simulation procedure. While the goal of the present study is to understand misfit relaxation in films grown at fairly low temperatures, the actual temperature used during the simulation is considerably higher. This was done because computational limitations force us to use a deposition rate that is very high compared to experimental conditions (as is the case in nearly all MD simulation studies of film growth). By carrying out the deposition at an elevated temperature, we artificially raise the surface diffusivity to the value it would have in low temperature depositions at experimentally accessible deposition rates. This can be done because the surface diffusion length increases exponentially with increasing temperature and decreases as the inverse square root of the deposition rate. Therefore, doubling the temperature can ameliorate the effects of a deposition rate which is orders of magnitude too large.

In Sec. II, we provide a detailed description of the simulation procedure employed. Next, we present the results of the growth of misfitting films on a finite temperature substrate. We start with a general analysis of the observed defect patterns and then compare the observed critical thicknesses with those predicted using a continuum, thermodynamic procedure and with energy minimizations of idealized films using the same interatomic potentials. We subsequently analyze the mechanisms of misfit dislocation nucleation, and 
finally we discuss the difference between the critical thicknesses observed in the cases of compressive and tensile misfit.

\section{METHOD}

Nonequilibrium MD simulations were performed with a technique previously developed for studies of film growth. ${ }^{17-20}$ The two-dimensional simulation cell is periodic in the $X$ direction and open in the $+Y$ direction (Fig. 1), with a width of $40 d_{0}$, where $d_{0}$ is the nearest neighbor spacing in a perfect triangular lattice using the interatomic potentials described below. The substrate (at the bottom of the simulation cell in Fig. 1) is 5 atomic layers thick, with the bottom layer frozen and with the next 2 layers (initially) thermostated at a predetermined temperature. This creates an isothermal substrate of essentially infinite thickness. Atoms are deposited at normal incidence to the substrate from a source which is effectively infinitely far above the growing film. As further explained below, the number of thermostated layers grows as the deposited film becomes increasingly thick.

The pair potential $U\left(r_{i j}\right)$ describing the interactions between two atoms $i$ and $j$ separated by a distance $r_{i j}$ is given by a Lennard-Jones potential that is modified to insure that the potential and its first derivative go to zero at a predetermined cut-off distance $r_{c}=2.1 r_{0}$ :

$$
U\left(r_{i j}\right)=\left\{\begin{array}{ll}
\epsilon\left[\left(\frac{r_{0}}{r_{i j}}\right)^{12}-2\left(\frac{r_{0}}{r_{i j}}\right)^{6}\right] & r_{i j} \leqslant r_{0} \\
\epsilon\left[\left(\frac{r_{0}}{r_{i j}}\right)^{12}-2\left(\frac{r_{0}}{r_{i j}}\right)^{6}\right]\left[3\left(\frac{r_{c}-r_{i j}}{r_{c}-r_{0}}\right)^{2}-2\left(\frac{r_{c}-r_{i j}}{r_{c}-r_{0}}\right)^{3}\right] & r_{i j}>r_{0}
\end{array} .\right.
$$

This modification only affects the potential for $r_{i j}>r_{0}$ (with $\left.r_{0} \approx d_{0}\right)$. The well depth $\epsilon$ is the same for all interactions (substrate-substrate, substrate-film, and film-film). The size parameter $\left(r_{0}\right)_{s}$ for the substrate-substrate interactions is kept fixed while that for the film-film interactions $\left(r_{0}\right)_{f}$ is varied so as to yield the desired misfit $f$ :

$$
f \equiv \frac{\left(r_{0}\right)_{f}-\left(r_{0}\right)_{s}}{\left(r_{0}\right)_{s}} .
$$

According to this definition, positive mismatch corresponds to the case in which the film atoms are larger than the substrate atoms (this will put the film into compression if the interfacial atoms are in perfect registry-no dislocations). Similarly, negative mismatch corresponds to the case in which the film is in tension (if film and substrate are in registry). The size parameter for the substrate-film interactions is taken as the algebraic mean of the substrate and film values.

In order to simplify the energy and distance scales reported here, we set the substrate parameters $\left(r_{0}\right)_{s}$ and $\epsilon$ and the mass of all atoms $M$ to unity. The corresponding reduced unit of time is $\tau=\sqrt{M\left(r_{0}\right)_{s}^{2} / \epsilon}$. The equations of motion are integrated with a variable time step scheme using the Nordsieck algorithm. ${ }^{18}$ The time step size is determined in terms of the maximum velocity at each time step, $\Delta t$ $=d_{0} /\left(200 V_{\text {max }}\right)$ so that no film atom moves more than $\approx 0.5 \%$ of the equilibrium interatomic spacing $d_{0}$ in any single step.

As the film growth proceeds, an increasing number of substrate and film layers are coupled to a heat bath, using the velocity scaling method of Berendsen et al. ${ }^{21}$ with a delay time of $0.1 \tau$. For thick films, 15 atomic layers remain unthermostated so that the atomic dynamics at the growth front remains unaffected. By including the atoms of the growing film in the thermostating, we prevent excessive heating dur- ing the deposition. This is important because the simulation would otherwise not account for the electronic contribution to the thermal conductivity (which usually dominates the phonon contribution in noninsulators). The temperature of the substrate and film is $0.175 \epsilon / k_{B}$. This is a relatively high temperature, i.e., of order half the melting temperature $\left[T_{m}\right.$ $=0.415 \epsilon / k_{B}$ (Ref. 22) of a pure two-dimensional LennardJones solid]. The positions of the frozen atoms at the bottom of the substrate correspond to equilibrium positions in a zero-pressure bulk material at the specified temperature. Since the substrate is modeled as if it were semi-infinite, no net expansion of the cell in the $X$ direction is allowed and

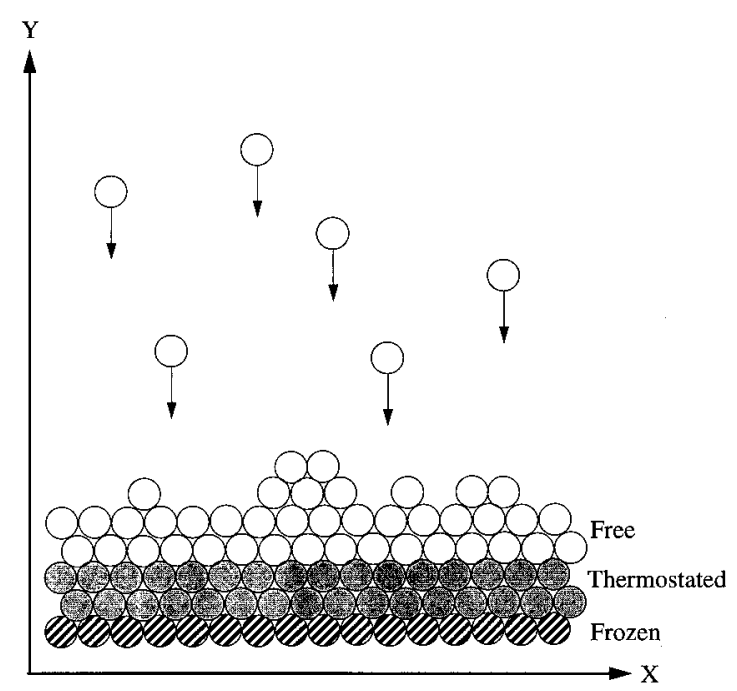

FIG. 1. Computational cell depicting the substrate, the growing film, and atoms impinging on the surface. The bottom layer of the substrate is frozen, the two layers above it are thermostated, and the atoms in subsequent layers are unconstrained. The thickness of the thermostated region increases as the film grows. 
this dimension is set in accordance with the thermostated temperature.

Incident atoms are deposited with a kinetic energy of $E_{b}=0.8 \mathrm{\epsilon} /$ atom and at a rate of 1 atom per $7.5 \tau$. As pointed out above, this deposition rate is very high compared to experimental conditions and would not allow for sufficient surface diffusion as compared with that during growth under common low temperature deposition conditions. The simulations are performed, however, with deposition onto a substrate with an artificially high temperature which ameliorates the effects of the unphysically large deposition rate. We also note that shock wave propagation caused by the impact of newly deposited material can be ignored because of the relatively low kinetic energy of the incoming atoms.

Critical thickness values are estimated by visual inspection of the atomic configurations during the course of the deposition. The existence of dislocations is confirmed by performance of a Burgers circuit. The reported errors in the critical thickness are obtained as the standard deviation of the results from five individual simulations. For comparison, the critical thicknesses are also calculated from a purely energetic standpoint. First, flat films are stretched/compressed into coherency with the substrate and the equilibrium atomic positions and the total energy are determined by energy minimization. For the latter, a Polak-Ribière conjugate gradient method was used, ${ }^{23}$ with a change in the total energy of $<10^{-7}$ as the convergence criterion. This process is then repeated without initially straining the film to coherency, resulting in a dislocated interface. By varying the number of film layers for a given misfit, the critical thickness is determined as that thickness for which the energy of the coherent and dislocated systems are identical. In order to be consistent with the corresponding dynamics data, it is important that the energy minimization is carried out at a slightly increased substrate density, namely the one that is appropriate for zeropressure conditions of the bulk material at zero temperature.

\section{DEFECTS AND STRESS RELAXATION}

We begin by examining the atomic structure of several films with different degrees of misfit. Figure 2 shows an unstrained film grown at $T=0.175 \epsilon / k_{B}$ with atoms deposited at an energy of $0.8 \mathrm{\epsilon}$ /atom. The top edge of the substrate is indicated by the two horizontal bars. Figure 2 shows a film with a surface roughness of $\pm 5 d_{0}$ that contains a small void. This surface roughness is typical of films grown under these conditions. In order to aid in the identification of defects, we shade the atoms in accordance with their atomic-level pressure. In all of the pressure plots, the indicated intermediate gray level corresponds to zero pressure and darker and lighter shading correspond to compression and tension, respectively. Examination of the shading around the void in Fig. 2 shows that there is compression below the void and tension above it. This is exactly the pattern of stress expected for an edge dislocation with a Burgers vector directed parallel to the substrate. Performing a Burgers circuit around the void (as shown in Fig. 2), indicates that there is indeed an edge dislocation with a Burgers vector parallel to the interface and directed from left to right in the figure, using the SF/RH Burgers circuit convention (see Ref. 24 for details).

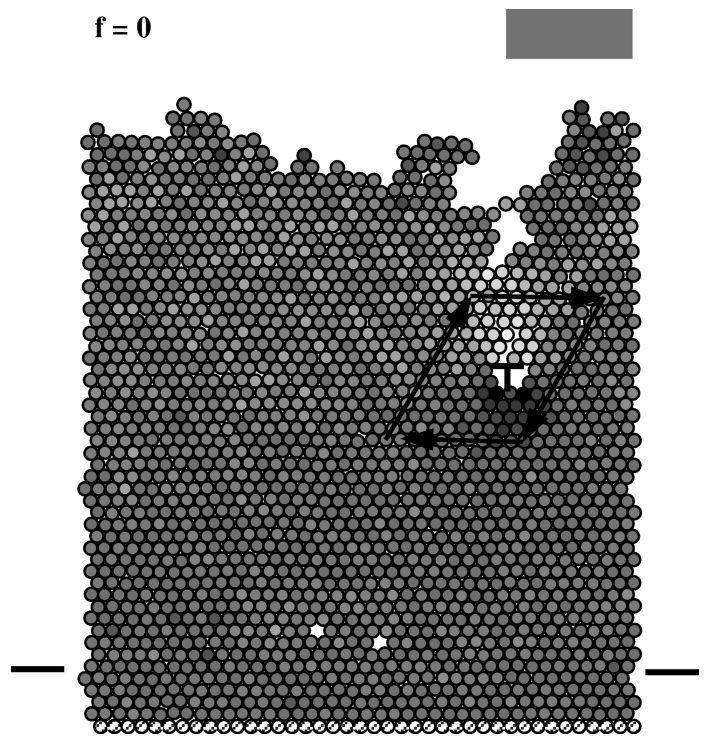

FIG. 2. Typical microstructure of an unstrained film. The frozen bottom layer of substrate atoms is shown as hatched; the two horizontal bars indicate the top edge of the substrate. The shading of the atoms represents the stress level, with light and dark shading indicating tension and compression, respectively. The shading corresponding to zero stress is indicated by the shaded bar above the figure.

Therefore, both the Burgers circuit and stress distribution demonstrate that there is an edge dislocation with its core centered inside the void in Fig. 2. Such dislocation/void aggregates are fairly common and the mechanism for their formation is associated with the presence of surface roughness during film growth. ${ }^{18}$

Figures 3(a)-3(c) show the atomic structure of films grown with compressive misfit strains corresponding to $f$ $=+5.0 \%$, $+2.5 \%$, and $+1.0 \%$, respectively. For the film structure at $f=5.0 \%$ [Fig. 3(a)], two misfit dislocations are observed at the film/substrate interface. Both dislocations have Burgers vectors parallel to the substrate (pointing toward the right), as seen either by the pressure distribution map or Burgers circuit. The substrate in Fig. 3(a) is $40 d_{0}$ wide, so that a misfit of $f=+5.0 \%$ implies that there are two fewer atoms in the atomic plane immediately above the interface as compared with below the interface. Therefore, two edge dislocations at the interface with Burgers vectors parallel to the interface and of length $d_{0}$ exactly cancel the long range misfit strain. These dislocations form very early in the course of the film growth simulation (see below).

Only one misfit dislocation is observed to form in the film grown with $f=+2.5 \%$ [Fig. 3(b)]. This dislocation has the same Burgers vector as those observed in the $f$ $=+5.0 \%$ simulation. However, unlike in the $f=+5.0 \%$ case where the dislocations sat directly at the interface, the dislocation here sits two atomic planes above the interface. For $f=+2.5 \%$ and a $40 d_{0}$ substrate, the long range part of the misfit strain is exactly cancelled by a single dislocation. In Fig. 3(c) $(f=+1 \%)$ no misfit dislocations are observed near the interface. This is not too surprising since introduction of a dislocation in a film with a misfit of $1 \%$ on a $40 d_{0}$ substrate (with periodic boundary conditions) would induce a strain larger and of opposite sign to the one that it would 


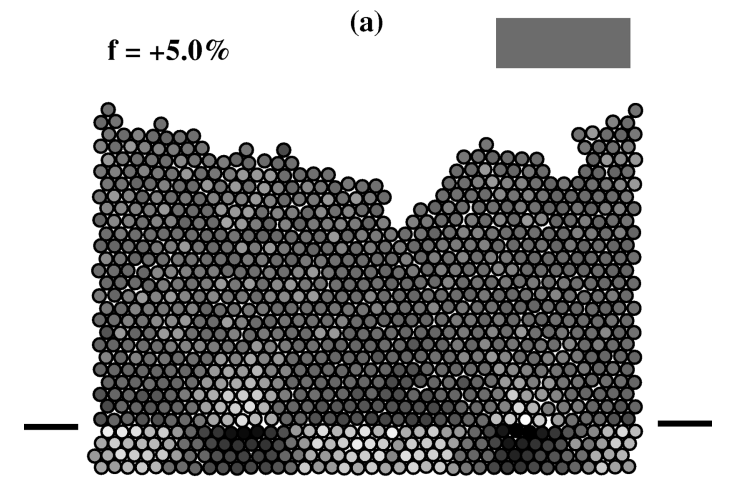

(b)

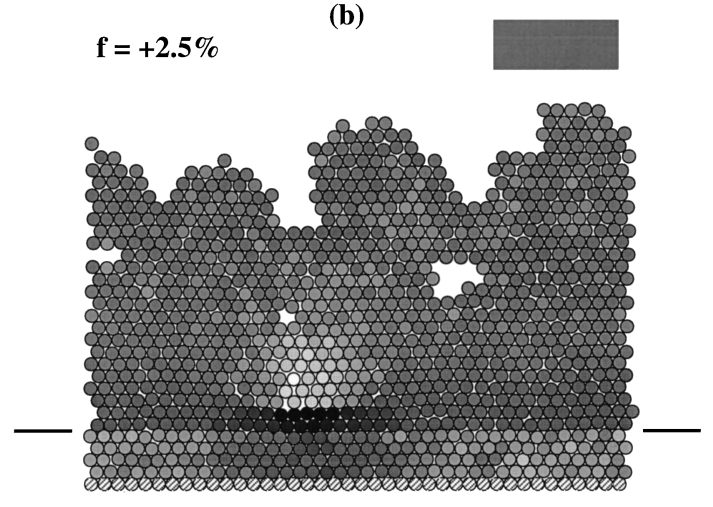

(c)

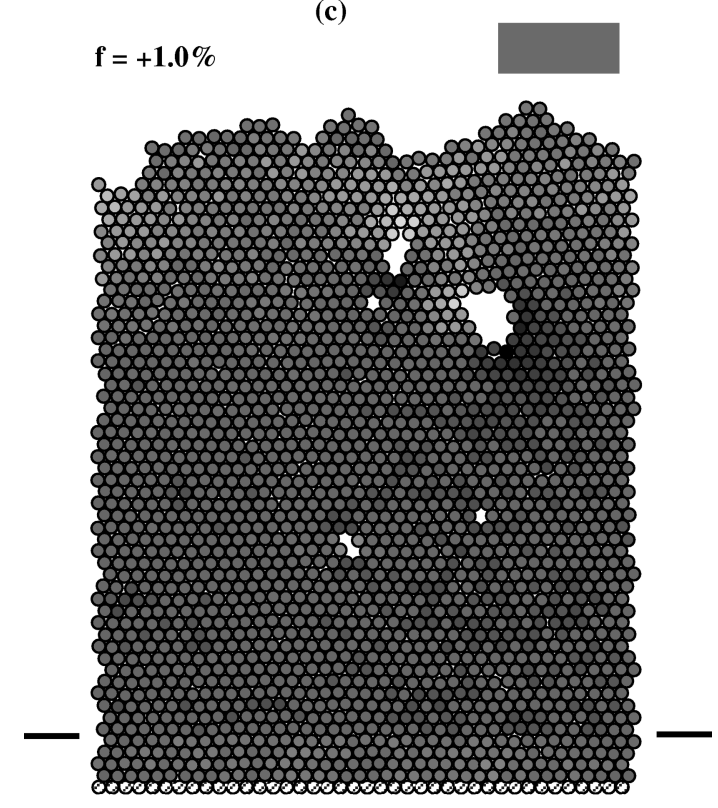

FIG. 3. Typical microstructures of films with positive misfits (= compressive stress in the film if in registry).

relieve. For this misfit, a substrate of at least $100 d_{0}$ would be required to make it thermodynamically favorable to introduce a dislocation. Several voids are observed to form in this $f=+1 \%$ film away from the interface. As shown in Fig. 2, such voids may naturally form during the growth process. The two voids closest to the surface contain edge dislocations: one with the Burgers vector canted by $60^{\circ}$ from the substrate and one parallel to the substrate. These dislocations likely form as a result of the growth process itself, rather than due to the misfit (see Fig. 2), although these dislocations (a)

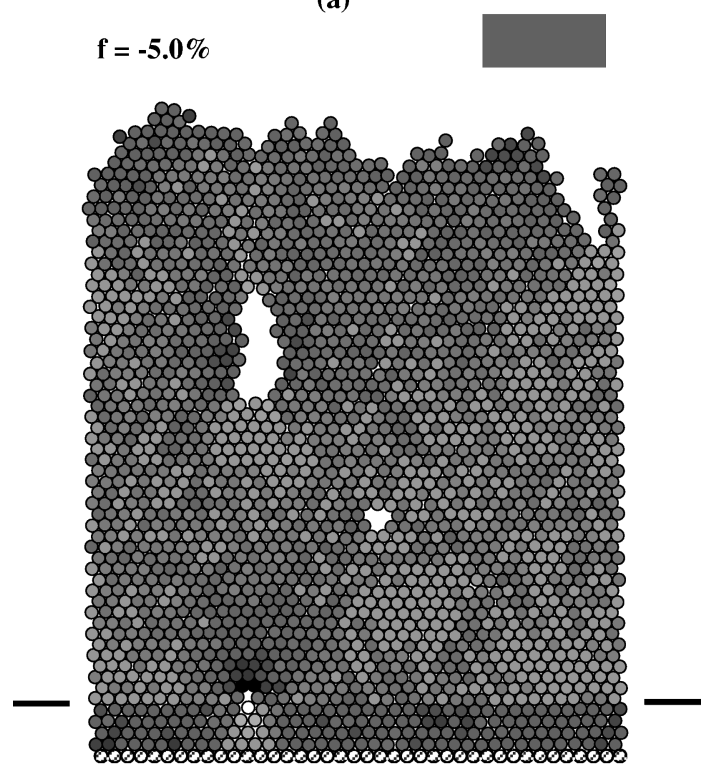

(b)

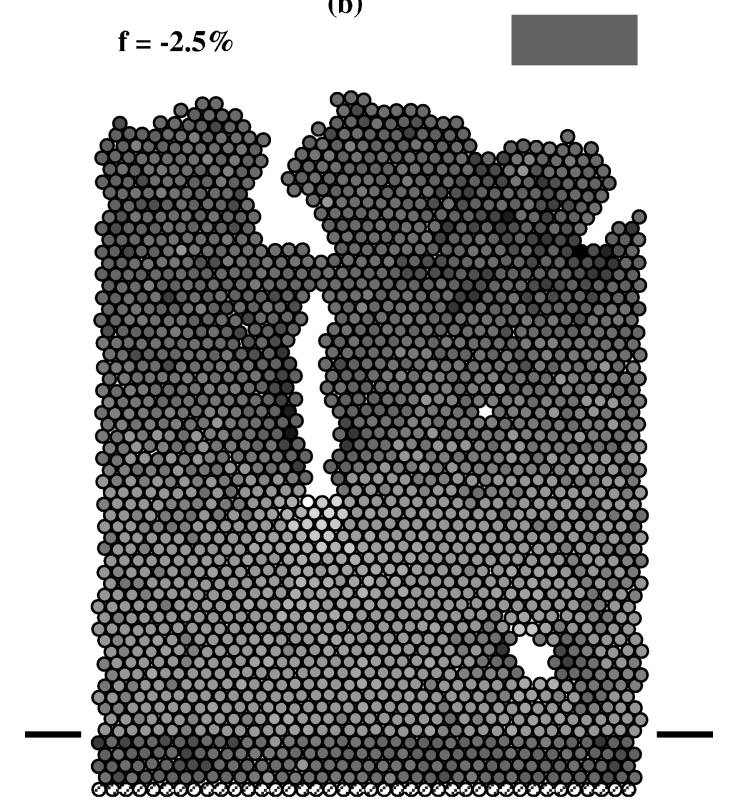

FIG. 4. Typical microstructures of films with negative misfits (=tensile stress in the film if in registry).

each have Burgers vectors which oppose the misfit strain.

Figures 4(a) and 4(b) show film microstructures for films grown with tensile misfit strains corresponding to $f$ $=-5.0 \%$ and $-2.5 \%$, respectively. In the $f=-5.0 \%$ case, a single edge dislocation is observed to form at the interface with a Burgers vector equal and opposite to those observed in the $f=+5.0 \%$ and $+2.5 \%$ films [see Figs. 3(a) and 3(b)]. The change in the sign of the Burgers vector is consistent with the change in the sign of the misfit. However, contrary to the $f=+5.0 \%$ case where two dislocations were observed to form at the interface, only one dislocation forms at the interface in this $f=-5.0 \%$ simulation even though the film is considerably thicker than its equilibrium critical thickness (see below). Therefore, the misfit strain is only partially (half) relieved by the dislocation formed. This can be seen by 


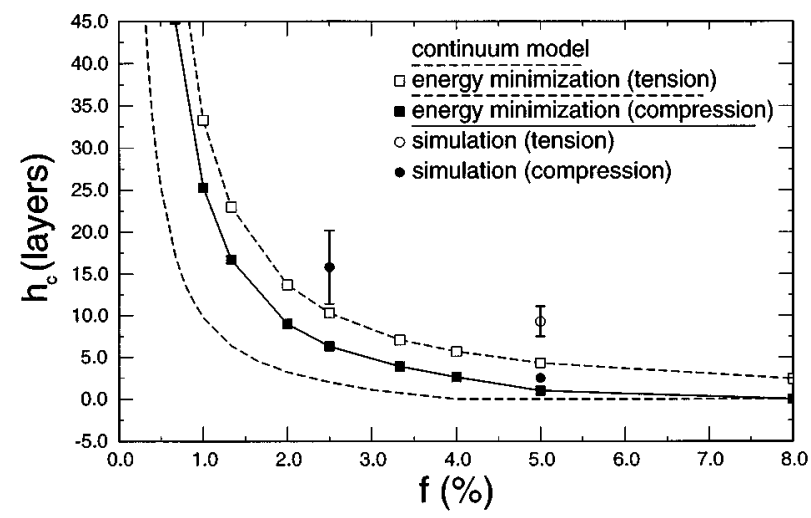

FIG. 5. Critical thickness as a function of the misfit. The dashed curve is obtained by energy minimization with a continuum model and applicable to both kinds of misfit. The solid and dotted curves are obtained by energy minimization of the atomistic model for positive (compressive) and negative (tensile) misfits, respectively. The solid circles are the MD simulation results for positive (compressive) misfits of $f=+2.5 \%$ and $f=+5.0 \%$, and the open circle is the simulation result for a negative (tensile) misfit of $f$ $=-5.0 \%$. (The error bar for the $5 \%$ compressive misfit case is smaller than the symbol size.)

the fact that the hydrostatic stress on the atoms above the dislocation is, on average, tensile [i.e., lighter in color than the background in Fig. 4(a)]. A sizable void is observed to form in this film away from the substrate. While this void contains no dislocations, the average tensile hydrostatic stress is lower near and above the void (the atoms are shaded darker) than below. This is likely attributable to the fact that the free surfaces of the void and those associated with the surface roughness provide some stress relief, as discussed below. In the $f=-2.5 \%$ case [Fig. 4(b)], no dislocations form at the film-substrate interface, even though the film is considerably thicker than its equilibrium critical thickness (see below). Two undislocated voids are observed within this structure. The upper void is greatly elongated in such a way as to maximize the amount of stress relief it can provide (i.e., in the direction normal to the substrate). The stresses near and above the elongated void are considerably less tensile than below the void. We analyze this effect in detail below.

In summary, we find that dislocations appear to nucleate more easily for the case of compressive misfit as compared to tensile misfit, and that voids can play a significant role in stress relaxation both in their dislocated and undislocated form.

\section{CRITICAL THICKNESS}

In order to determine the critical thickness as a function of misfit strain, we performed a series of MD simulations of film growth and measured the film thickness at which dislocations first appear. In this way, the critical thickness determination includes all aspects of the growth morphology, atomic vibrations and detailed dislocation nucleation mechanisms. We performed such simulations for $f= \pm 5.0 \%$ and $\pm 2.5 \%$, using a substrate of width $40\left(d_{0}\right)_{s}$. No dislocations were observed near the substrate for $f=-2.5 \%$. The critical thicknesses $h_{c}$ for the remaining three films are plotted in Fig. 5 as a function of the misfit. These data show that the critical thickness increases with decreasing misfit strain and is larger in tension than in compression. The fact that no dislocations were observed for the $f=-2.5 \%$ films suggests that they have a very large critical thickness. The dependence of the critical thickness on the sign of the misfit is unexpected on the basis of the continuum theories for the critical thickness.

In order to evaluate the magnitude of the critical thicknesses obtained from the simulations, we compare the simulation results with the predictions of the equilibrium, continuum theory. To do this, we follow the approach of Matthews and Blakeslee ${ }^{2}$ (corrected for a single array of dislocations in two dimensions):

$$
h_{c}=\frac{b}{8 \pi(1+v) f}\left[\ln \left(\frac{h_{c}}{b}\right)+1\right] .
$$

Using $v=1 / 3$ (a rigorous result for a two-dimensional triangular, nearest neighbor, central force model), ${ }^{25}$ we numerically solve for the critical thickness using the implicity expression in Eq. (3). This theoretical prediction of the critical thickness is plotted as a function of $f$ in Fig. 5. In all cases, we see that the predicted critical thickness is less than that obtained from the simulations.

One possible source of the discrepancy between the atomistic simulation results and the continuum theory [Eq. (3)] is associated with the assumption that classical linear elasticity describes the atomic interactions. In reality, the interatomic potential is not exactly harmonic: the potential is stiffer in compression than in tension. A second possible source of error is associated with the dislocation nucleation mechanism, which the continuum theory does not account for. In order to separate these two effects, we perform a thermodynamic analysis of the critical thickness using the same potentials employed in the MD simulations. To this end, we explicitly minimized the total potential energy both in the presence and in the absence of misfit dislocations, starting out from an ideal thin film geometry (i.e., perfectly flat surfaces). The energy of the system with disregistry increases more slowly with increasing film thickness than the energy of the corresponding coherent system; the critical thickness is the particular number of epilayers for which the two energies become equal. The results are also shown in Fig. 5, for both compressive and tensile misfits.

It can be seen that the critical thickness values are not only increased over the linear, continuum elasticity predictions, but that, on purely energetical grounds, there is a significant difference between the compressive and tensile cases. The difference in the absolute magnitude between the energy minimization results and continuum theory results may, in part, be attributable to assumptions made in the theory regarding the dislocation core energy. The difference between the compressive and tensile misfit cases, on the other hand, can only be attributed to the anharmonicity of the pair potential. The anharmonicity of any Lennard-Jonestype potential is such that the strain energy due to disregistry is larger for a compressive distortion than for a corresponding tensile distortion. For a mismatch of $|f|=5.0 \%$, e.g., the stored energy due to uniaxial strain is $0.120 \mathrm{\epsilon} / \mathrm{atom}$ in the compressive case, but only $0.077 \mathrm{\epsilon}$ /atom in the tensile case 
(a)

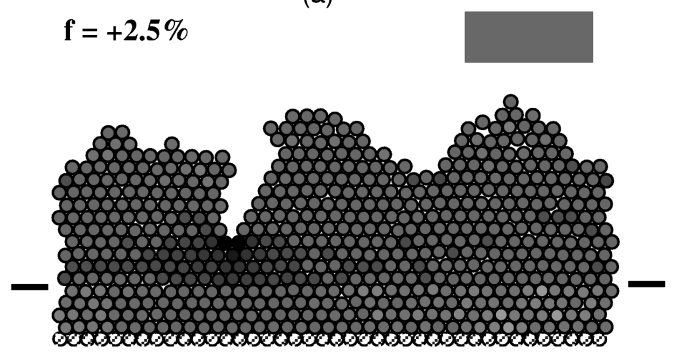

(b)

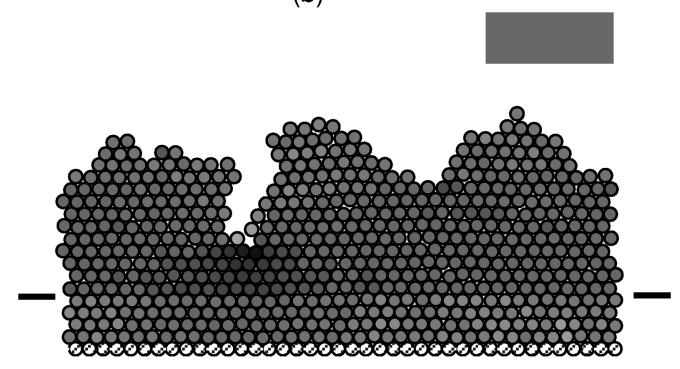

(c)

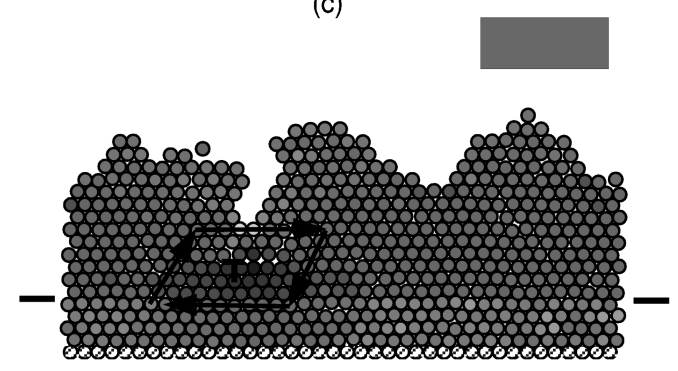

(d)

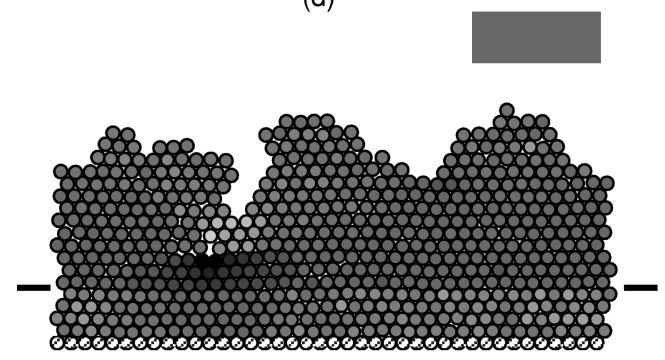

(e)

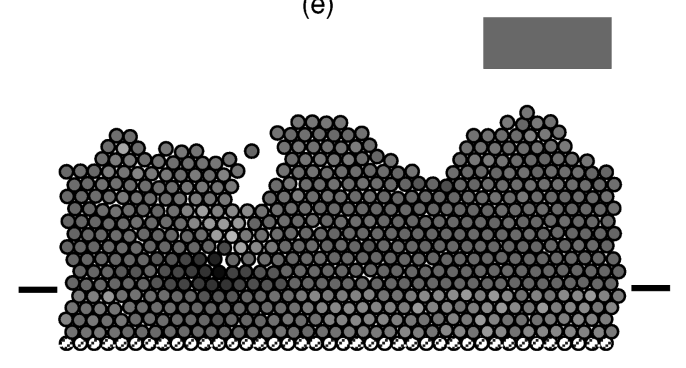

(f)

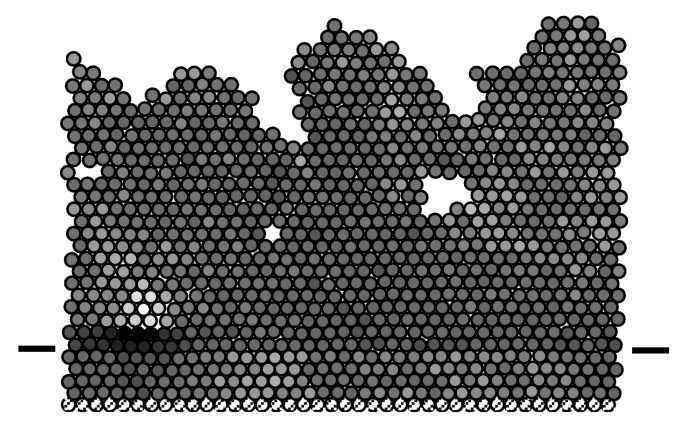

FIG. 6. Time sequence illustrating misfit dislocation nucleation for a film with a positive (compressive) misfit of $f=+2.5 \%$.

(a difference of order 50\%); for a mismatch of $|f|=2.5 \%$, the same figures are $0.026 \epsilon$ and $0.021 \epsilon$, respectively (a difference of order 20\%). Examination of Fig. 5 shows that in the tensile case energy minimization results lead to an underprediction of the critical thickness. A similar effect is seen in the compressive case for $f=2.5 \%$, while there is better agreement at $f=5.0 \%$. These disagreements suggest that the dislocation nucleation mechanism and/or the nonflat nature of the MD grown film play significant roles in determining the critical thickness.

\section{MISFIT DISLOCATION NUCLEATION MECHANISMS}

By analyzing the atomic configuration at short time intervals during film growth, we are able to identify the detailed dislocation nucleation mechanisms in all of the simulations. Figure 6 shows a typical growth sequence for an $f$ $=+2.5 \%$ film. In this case, the film develops a nonflat surface morphology prior to achieving the critical thickness [Fig. 6(a)]. Because of the compressive stress in the film, a highly compressive region develops at the bottom of the largest surface depression (see Sec. VI for more details). In order to relax the high stress concentration at the bottom of the most prominent depression, the atoms adjust their po- sitions and the plane (line) of atoms at the bottom of the depression effectively buckles, ejecting an atom into the plane above it [cf. Figs. 6(b) and 6(c)]. Removing this highly compressed atom in this way leaves the bottom of the surface depression in tension [Fig. 6(c)]. Associated with this buckling event is the formation of an edge dislocation with a Burgers vector parallel to the substrate (oriented so as to relieve the misfit). This is confirmed by drawing a Burgers circuit in Fig. 6(c) and by the characteristic stress couple seen in Fig. 6(d). To further relax the misfit strain, the dislocation climbs downwards, toward the substrate, further relieving the strain [Figs. 6(e) and 6(f)]. Figure 6(f) also shows that the dislocation is free to glide parallel to its Burgers vector. Comparison of Figs. 6(a) and 6(f) also shows that the formation of the dislocation reduces the stress within the film.

The sequence of events shown in Fig. 6 was observed to occur in most of our simulations of compressively strained films that form dislocations. Therefore, we conclude that under compressive misfit conditions, dislocations nucleate in regions of very large compressive stress at the base of surface depressions via a single-atom buckling mechanism. Subsequent nonconservative and conservative motion of the 


\section{$f=-\mathbf{5 . 0 \%}$}

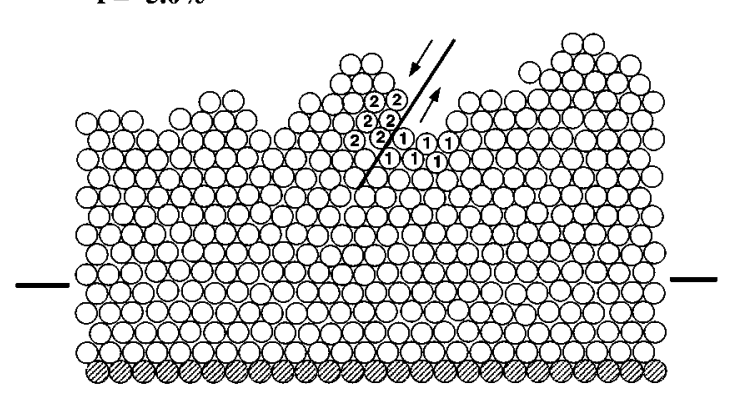

(b)

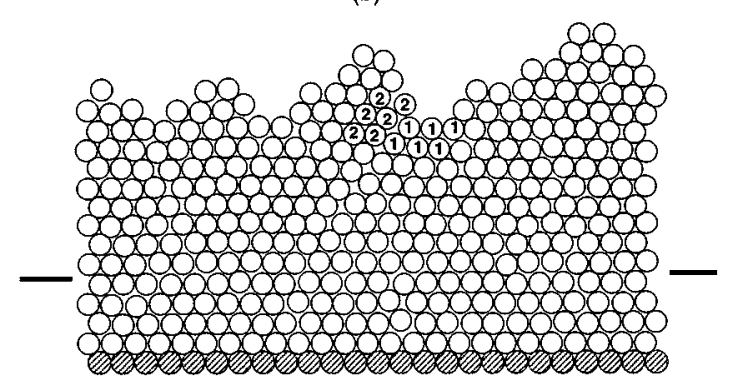

(c)

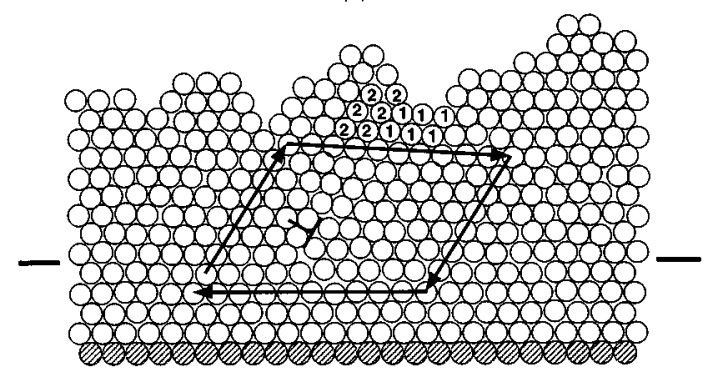

(d)

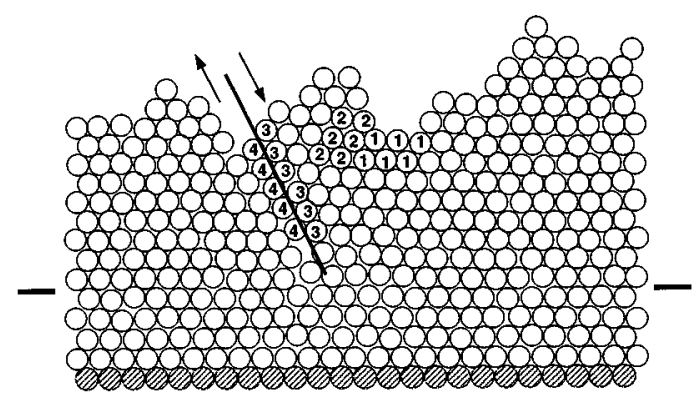

(e)

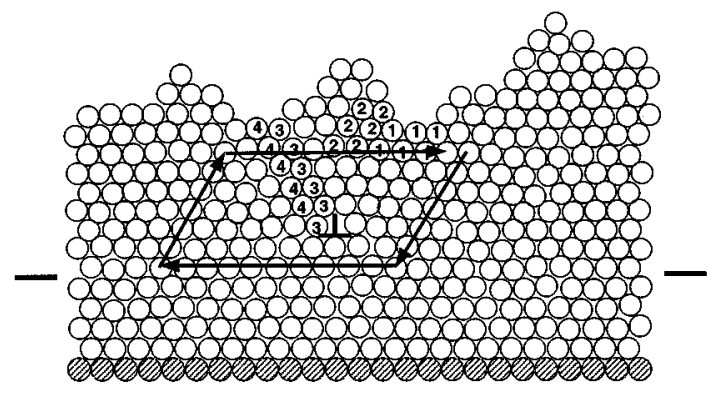

FIG. 7. Time sequence illustrating misfit dislocation nucleation for a film with a negative (tensile) misfit of $f=-5.0 \%$.

dislocation maximizes the amount of stress relief within the structure. This usually corresponds to climbing down towards the interface and gliding away from the surface depression.

Interestingly, we find that the mechanism of dislocation nucleation is very different in the case of initial tensile stress. Figure 7 illustrates a sequence of structures observed during film growth for a mismatch of $f=-5.0 \%$. Stress levels are not shown because the different images were captured at very short time increments, such that our usual procedure of averaging the stress over the time span between images is not sufficient to smear out the effects of thermal vibrations. Figure 7(a) shows the film structure immediately before the nucleation of misfit dislocations. Two groups of atoms close to the surface are observed to slide relative to one another [as indicated in Fig. 7(a)], resulting in the atomic configuration shown in Fig. 7(b). The Burgers vector of the dislocation formed in this way is not parallel to the interface [Fig. 7(c)], and thus only part of the Burgers vector contributes to the strain relaxation. Following the nucleation of this fully formed dislocation, another two groups of atoms [" 3 "' and ' 4 "' in Fig. 7(d)] slide relative to each other [Fig. 7(d)]. This creates an additional dislocation with a Burgers vector which is at a $60^{\circ}$ angle from that of the dislocation formed earlier.
The two dislocations are attracted toward each other and glide together, reacting to form a single dislocation with a Burgers vector that is parallel to the interface [Fig. 7(e)]. Examination of the stress field produced by the first dislocation shows that the position where the new dislocation forms corresponds to a maximum in the attractive Peach-Koehler force between the two dislocations (for any point along the surface). This provides an explanation as to the location where the second dislocation is formed [Fig. 7(e)]. Figure 4(a) shows the atomic configuration of this growing film at much later time.

This dislocation nucleation mechanism has also been observed in many of our simulations where the film grows with a tensile misfit. In these cases, misfit dislocations are formed by coordinated sliding events that produce two dislocations with Burgers vectors which are not parallel to the substrate, but which react to form a single dislocation with a Burgers vector oriented parallel to the substrate.

\section{DISCUSSION}

The as-grown film structures are often substantially different from that commonly assumed in the classical, continuum analyses of the critical thickness. The two main non- 
idealities are film roughness and void formation. Surface roughness and voids are both common features of films grown at low temperature (whether by molecular beam epitaxy, magnetron sputtering, or other techniques), even in the absence of stresses generated by misfit. ${ }^{18,20,26-31}$ The presence of stresses within the film can modify the thermodynamics and kinetics of the formation of both surface roughness and voids. Several theoretical and experimental studies have shown that both tensile and compressive stresses can lead to the development of a surface instability that tends to roughen the surface (see e.g., Refs. 32 and 33).

Once voids or surface roughness develop, they modify the distribution of stresses within the film. Consider the case of the elongated void seen in the tensile films shown in Figs. 4(a) and 4(b). The stress map indicates that the stresses are more tensile near the bottom and top surfaces of the voids and that there is a compressive stress near the sides. This can be understood by reference to the elasticity solution for an elliptical cylindrical void of semiaxes $a$ and $b$ [ellipticity $m=(a-b) /(a+b) ; m=0$ is a circle and $m= \pm 1$ is a crack] with a uniaxial stress $\sigma_{0}$ applied parallel to semiaxis $b .^{34}$ The tangential stress $\sigma_{\tau \tau}$ around the elliptical hole is given by

$$
\begin{aligned}
& \sigma_{\tau \tau}(\theta)=\sigma_{0}\left[\frac{1-2 m-m^{2}+2 \cos (2 \theta)}{1+m^{2}-2 m \cos (2 \theta)}\right], \\
& \sigma_{\tau \tau}(0)=\sigma_{0}\left[\frac{3+m}{1-m}\right] \\
& \sigma_{\tau \tau}\left(\frac{\pi}{2}\right)=-\sigma_{0},
\end{aligned}
$$

where $\theta$ is measured with respect to semiaxis $a . \sigma_{\tau \tau}(\pi / 2)$ corresponds to the sides of the voids, and hence should show a stress opposite to that of the applied stress, as seen in Figs. 4(a) and 4(b). $\sigma_{\tau \tau}(0)$ corresponds to the bottom and top edges of the void, and hence the stress there should have the same sign as the applied stress but of greater magnitude. This too is consistent with the stresses shown around the voids in Figs. 4(a) and 4(b). Equation (4) shows that the magnitude of the stress at the top and bottom of the elongated void gets larger with increasing eccentricity, $m$. While the stress can get very large at the top and bottom of the void, the presence of the free surfaces of the void that are perpendicular to the substrate provide very effective stress relief over a distance comparable to the height of the void.

Elastic analyses of surface roughness also demonstrate that these features can both lead to very large localized stresses and overall stress relaxation. The stress along a sinusoidal surface profile of amplitude $A$ and wavelength $\lambda$ in a body loaded by an in-plane stress $\sigma_{0}$ is (see e.g., Refs. 33 and 35)

$$
\sigma_{\tau \tau}(x)=\sigma_{0}\left[1+\frac{4 \pi A}{\lambda} \cos \left(\frac{2 \pi x}{\lambda}\right)\right] .
$$

This expression shows that there is a stress concentration of magnitude $[1+(4 \pi A) / \lambda]$ at the bottom of the valleys and the stress decreases by $\sigma_{0} 4 \pi A / \lambda$ near the peaks. This is consistent with the type of stress concentration observed near the bottom of the valleys and near the peaks in Fig. 6(a). The stress concentration at the bottom of the valleys increases in direct proportion with the depth of the valleys.

Spatial variation in the stresses in misfitting, coherent films due to nonuniformities in their surface morphology can significantly modify the film thickness at which dislocation nucleation occurs. Jesson et al. suggested that the presence of deep valleys on the surface are the main sites for dislocation nucleation during heteroepitaxy $\mathrm{y}^{36}$ and that these valleys form spontaneously due to the heteroepitaxial stresses, as described above. The formation of such valley structures (and/or elongated voids) and the preferential nucleation of dislocations at the bottom of these valleys was repeatedly observed in the present simulations for films that were either in compression or tension, in agreement with these predictions. The important conclusion is that the surface morphology that gives rise to preferential (heterogeneous) nucleation of dislocations forms during the growth process and is dependent on the atomic details of the growth process and the film stresses. This complicates the analysis of the critical thickness because the film morphology that develops during film growth depends on the magnitude of the stresses (i.e., misfit) within the film. In fact, both linear and nonlinear analyses of the evolution of surface profiles show that flat surfaces are thermodynamically and kinetically unstable and can evolve into structures with deep, narrow (cracklike) valleys. $^{32,33,37-40}$ It is exactly this type of surface relief that can provide the large stress concentrations required for dislocation nucleation. ${ }^{36}$

In a perfect film with a flat surface, as assumed in the continuum analyses, dislocation nucleation would be extraordinarily difficult and the actual critical thickness would be very large. Zimmerman and $\mathrm{Gao}^{4}$ considered dislocation nucleation in an atomistic simulation of a material with a nearly flat surface (i.e., one with atomic steps). They found that a critical strain was required to nucleate a dislocation, nearly independent of film thickness. This is in contradiction with the theoretical concept of a critical thickness. This result suggests that the thermodynamic critical thickness will not be observed unless dislocations are already present or are very easily nucleated. If no dislocations are initially present, the actual critical thickness will likely be controlled by the ease of dislocation nucleation. Since dislocation nucleation depends on the structure and morphology of the film this critical thickness will likely vary from material-to-material even if the physical parameters (misfit, modulus, Burgers vector) are the same. This is consistent with our observations, which show that the the film morphology plays an important role in the dislocation nucleation mechanism.

Because of the idealized nature of the films grown in the present simulations (i.e., two-dimensional, no preexisting dislocations), the possible sources of dislocations are somewhat limited. Fitzgerald et $a l^{42}$ identified three distinct types of dislocation sources: fixed sources (including substrate dislocations and substrate surface inhomogeneities), dislocation multiplication, and surface half-loop nucleation. Each of these types of sources may be activated at distinct strain levels. ${ }^{33}$ Fixed sources are thought to have the lowest activation energy and are thus likely to be activated first. In line 
with Matthews and Blakeslee's theory of threading dislocations, ${ }^{2}$ fixed sources would therefore be responsible for the observed critical thickness. This type of dislocation source does not require dislocation nucleation at all, and hence should lead to results in reasonable agreement with the thermodynamic theory. In our simulations, dislocation nucleation by the two-dimensional analog of the half-loop mechanism occurs. This mechanism is enhanced by the stress concentration associated with the nonplanar surface morphology. Fixed sources are excluded from the discussion here because we are focussing on the formation of the first dislocation and not the formation of subsequent dislocations. Tersoff and LeGoues recently compared the kinetic barriers for dislocation nucleation by strain-induced surface roughening with nucleation from other sources. ${ }^{43}$ They found that the former is favored at "large" misfits, such as those considered in the present article. The validity of this conclusion was subsequently questioned by Christiansen et al. ${ }^{44}$ This issue clearly remains unresolved.

The thermodynamic predictions of the critical thickness for dislocation nucleation suggest that the same critical thickness should be observed for the same magnitude of misfit, independent of whether it is tensile or compressive. This is not consistent with our simulation results which show that the compressively strained film has a smaller critical thickness than a film with a tensile misfit of equal magnitude. As discussed above, this asymmetry may be attributed to the anharmonicity of the present (Lennard-Jones) interatomic potential. This is in agreement with the analytical work of Markov and Milchev. ${ }^{45}$ There may also be kinetic factors that can help rationalize this behavior on the basis of the observed dislocation nucleation mechanisms. In the compressive misfit case, the dislocations nucleate by squeezingout an atom at the base of surface depressions (see Fig. 6). This type of event occurs quite naturally during the deposition process. In the tensile misfit case, however, the nucleation of misfit dislocations involves the concerted motion of relatively large numbers of atoms, leading to insertion of an extra lattice (plane) row into an already continuous film. Accordingly, the kinetic barrier in the tensile misfit case is larger than in the compressive case. In fact, the barrier may be sufficiently large that it can only be overcome in two smaller steps. This would explain why the Burgers vector of the initially formed dislocation in a tensile film is not parallel to the interface. As described in the previous section, the Burgers vector only becomes parallel to the interface after a second collective atomic rearrangement step.

Our observation of a compression-tension asymmetry in the critical thickness is consistent with the recent quasistatic calculations of semiconductor heterostructures by Ichimura and Narayan, ${ }^{14,15}$ and the interpretation of a collection of experimental observations by Markov and Milchev ${ }^{45}$ for metal-on-metal growth. On the other hand, Wegscheider and Cerva $^{46}$ experimentally found that the critical thickness in the $\mathrm{Si} / \mathrm{SiGe}$ system is larger in the case of compressive misfit, rather than tensile misfit. This discrepancy with the simulation results could be indicative of a number of issues. First, our simulations are two-dimensional rather than threedimensional, thereby limiting the types of dislocations that can form in the present simulations to pure edges, while in $\mathrm{Si} / \mathrm{SiGe}$ the actual dislocations are of mixed type. Second, our simulations are based on close-packed systems (like metals) rather than the much more open (low coordination number) diamond cubic structure of the semiconductor.

\section{CONCLUSION}

The growth and relaxation of misfitting, coherent films was simulated using molecular dynamics in two dimensions with Lennard-Jones potentials. The goal of this study was to identify the mechanisms for misfit dislocation nucleation and stress relaxation in a well-defined model film-substrate system whose surface morphologies develop during film growth. Pseudomorphic film growth was observed up to a critical thickness. The formation of voids within the film was found to relax the overall stress level near the void while creating regions of stress concentrations near the void surface parallel to the substrate. At the critical thickness, dislocations formed, and relaxed most of the misfit strain. The critical thickness was shown to increase with decreasing lattice mismatch and was sensitive to the sign of the misfit, in contradiction with theoretical predictions. The critical thickness of compressively strained films was smaller than for films with the same magnitude of misfit, but in tension. The mechanism of dislocation nucleation was observed to be different in tension and compression and, in all cases, was associated with the roughness of the film surface. In the compressive misfit case, dislocations nucleated by squeezing-out an atom at the base of surface depressions. In the tensile misfit case, however, the nucleation of misfit dislocations involved the concerted motion of a relatively large number of atoms, leading to insertion of an extra lattice (plane) row into an already continuous film. The present results demonstrate that the dislocation nucleation mechanism is intimately associated with surface roughness and the stress concentration that it produces. Since the surface roughness develops as an integral part of the film growth process, it is not possible to separate stress relaxation via dislocation nucleation (and the critical thickness) from the film growth process itself. This suggests that careful control of the film morphology during growth can be used in order to obtain pseudomorphic films of greater thicknesses than predicted on the basis of thermodynamic analyses.

\section{ACKNOWLEDGMENTS}

This research was supported by the U.S. Advanced Projects Research Agency and the U.S. Air Force Office of Scientific Research under MURI Grant No. F49620-95-10524 .

\footnotetext{
${ }^{1}$ F. C. Frank and J. H. van der Merwe, Proc. R. Soc. London, Ser. A 198, 205 (1949); J. H. van der Merwe, J. Appl. Phys. 34, 123 (1963).

${ }^{2}$ J. W. Matthews and A. E. Blakeslee, J. Cryst. Growth 27, 118 (1974).

${ }^{3}$ J. W. Matthews, J. Vac. Sci. Technol. 12, 126 (1975).

${ }^{4}$ J. C. Bean, L. C. Feldman, A. T. Fiory, S. Nakahura, and I. K. Robinson, J. Vac. Sci. Technol. A 2, 436 (1984).

${ }^{5}$ P. J. Orders and B. F. Usher, Appl. Phys. Lett. 50, 980 (1987).

${ }^{6}$ I. J. Fritz, Appl. Phys. Lett. 51, 1080 (1987).

${ }^{7}$ R. People and J. C. Bean, Appl. Phys. Lett. 47, 322 (1985).

${ }^{8}$ B. W. Dodson and J. Y. Tsao, Appl. Phys. Lett. 51, 1325 (1987).
} 
${ }^{9}$ S. V. Kamat and J. P. Hirth, J. Appl. Phys. 67, 6844 (1990).

${ }^{10}$ B. W. Dodson and P. A. Taylor, Appl. Phys. Lett. 49, 642 (1986).

${ }^{11}$ M. H. Grabow and G. H. Gilmer, in Semiconductor-Based Heterostructure, edited by M. L. Green et al. (Metallurgical Society, Warrendale, PA, 1986).

${ }^{12}$ A. S. Nandedkar, Acta Metall. Mater. 41, 3455 (1993).

${ }^{13}$ G. Cohen-Solal, F. Bailly, and M. Barbe, J. Cryst. Growth 138, 68 (1994).

${ }^{14}$ M. Ichimura and J. Narayan, Philos. Mag. A 72, 281 (1995).

${ }^{15}$ M. Ichimura and J. Narayan, Mater. Sci. Eng. B 31, 299 (1995).

${ }^{16}$ P. A. Ashu, J. H. Jefferson, A. G. Cullis, W. E. Hagston, and C. R. Whitehouse, J. Cryst. Growth 150, 176 (1995).

${ }^{17}$ R. W. Smith and D. J. Srolovitz, in Evolution of Epitaxial Structure and Morphology, edited by A. Zangwill, D. Jesson, D. Chambliss, and R. Clarke (Materials Research Society, Pittsburgh, PA, 1996).

${ }^{18}$ R. W. Smith and D. J. Srolovitz, J. Appl. Phys. 79, 1448 (1996).

${ }^{19}$ F. Ying, R. W. Smith, and D. J. Srolovitz, Appl. Phys. Lett. 69, 3007 (1996).

${ }^{20}$ L. Dong, R. W. Smith, and D. J. Srolovitz, J. Appl. Phys. 80, 5682 (1996).

${ }^{21}$ H. J. C. Berendsen, J. P. M. Postma, W. F. Van Gunsteren, A. DiNola, and J. R. Haak, J. Chem. Phys. 81, 3684 (1984).

${ }^{22}$ F. F. Abraham, in Melting, Localization, and Chaos, edited by R. K. Kalia and P. Vashishta (Elsevier, New York, 1982).

${ }^{23}$ W. H. Press, S. A. Teukolsky, W. T. Vetterling, and B. P. Flannery, Numerical Recipes in $C$, 2nd ed. (Cambridge University Press, Cambridge, UK, 1992).

${ }^{24}$ J. P. Hirth and J. Lothe, Theory of Dislocations, 2nd ed. (Wiley, New York, 1982), p. 23.

${ }^{25}$ W. H. Yang, D. J. Srolovitz, G. N. Hassold, and M. P. Anderson, in Simulation and Theory of Evolving Microstructures, edited by M. P. Anderson and A. D. Rollett (Metallurgical Society, Warrendale, PA, 1990), p. 277.

${ }^{26}$ B. A. Movchan and A. V. Demchishin, Fiz. Met. Metalloved. 28, 83 (1969).
${ }^{27}$ J. A. Thornton, Annu. Rev. Mater. Sci. 7, 239 (1977).

${ }^{28}$ G. S. Bales, R. Bruinsma, E. A. Eklund, R. P. U. Karunasiri, J. Rudnick, and A. Zangwill, Science 249, 264 (1990).

${ }^{29}$ T. J. Vink, M. A. J. Somers, J. L. C. Daams, and A. G. Dirks, J. Appl. Phys. 70, 4301 (1991).

${ }^{30}$ T. D. Andreadis, M. Rosen, M. I. Haftel, and J. A. Sprague, Surf. Coat. Technol. 51, 328 (1992).

${ }^{31}$ M. Misheva, N. Djourelov, T. Kotlarova, D. Elenkov, and G. Passage, Thin Solid Films 283, 26 (1996).

${ }^{32}$ M. A. Grinfeld, Sov. Phys. Dokl. 31, 831 (1987).

${ }^{33}$ D. J. Srolovitz, Acta Metall. 37, 621 (1989).

${ }^{34}$ N. I. Muskhelishvili, Some Basic Problems of the Mathematical Theory of Elasticity, 4th ed. (Noordhoff, Leyden, 1977).

${ }^{35}$ H. Gao, J. Mech. Phys. Solids 39, 443 (1991).

${ }^{36}$ D. E. Jesson, S. J. Pennycook, J. M. Baribeau, and D. C. Houghton, Phys. Rev. Lett. 71, 1744 (1993).

${ }^{37}$ B. J. Spencer, P. W. Voorhees, and S. H. Davis, Phys. Rev. Lett. 67, 3696 (1991)

${ }^{38}$ C.-H. Chin and H. Gao, Int. J. Solids Struct. 30, 2983 (1993).

${ }^{39}$ W. H. Yang and D. J. Srolovitz, Phys. Rev. Lett. 71, 1593 (1993).

${ }^{40}$ W. H. Yang and D. J. Srolovitz, J. Mech. Phys. Solids 42, 1551 (1994).

${ }^{41}$ J. A. Zimmerman and H. Gao, in Evolution of Epitaxial Structure and Morphology, edited by A. Zangwill, D. Jesson, D. Chambliss, and R. Clarke (Materials Research Society, Pittsburgh, 1996).

${ }^{42}$ E. A. Fitzgerald, G. P. Watson, R. E. Proano, and D. G. Ast, J. Appl. Phys. 65, 2220 (1988).

${ }^{43}$ J. Tersoff and F. K. LeGoues, Phys. Rev. Lett. 72, 3570 (1994).

${ }^{44}$ S. Christiansen, M. Albrecht, H. Michler, and H. P. Strunk, in Strained Layer Epitaxy-Materials, Processing, and Device Applications, edited by E. A. Fitzgerald, J. Hoyt, K.-Y. Cheng, and J. Bean (Materials Research Society, Pittsburgh, 1995).

${ }^{45}$ I. Markov and A. Milchev, Surf. Sci. 136, 519 (1984); 145, 313 (1984).

${ }^{46}$ W. Wegscheider and H. Cerva, J. Vac. Sci. Technol. B 11, 1056 (1993). 